Check for updates

Cite this: Chem. Commun., 2022, 58,689

Received 23rd September 2021 Accepted 22nd November 2021

DOI: $10.1039 / \mathrm{d} 1 \mathrm{cc} 05350 \mathrm{~g}$

rsc.li/chemcomm

\section{Blatter-type radicals as polarizing agents for electrochemical overhauser dynamic nuclear polarization $\dagger$}

\author{
Felipe Saenz, (D) $\ddagger^{*^{a}}$ Mika Tamski, (D) $\ddagger^{*^{a}}$ Jonas Milani, (D) $\ddagger^{*^{a}}$ Christophe Roussel, (D) ab \\ Holger Frauenrath (D) ${ }^{c}$ and Jean-Philippe Ansermet (D) ${ }^{a}$
}

\begin{abstract}
Overhauser dynamic nuclear polarization (O-DNP) refers to a microwave-assisted process where an unpaired electron's (e.g. a radical) spin polarization is transferred to surrounding nuclei in solution, thus increasing the nuclear magnetic resonance (NMR) signal intensity of a given substance by several orders of magnitude. The presence of the unpaired electrons, which induces relaxation of the resulting hyperpolarized state when the radiation is halted, can be avoided by electrochemically removing the radicals on demand. We report the use of Blatter-type (benzo[e][1,2,4]triazinyl) radicals as polarizing agents, potentially opening the way to highly tunable radicals for electrochemical DNP.
\end{abstract}

Nuclear magnetic resonance (NMR) spectroscopy and magnetic resonance imaging (MRI) are powerful and robust characterization methods widely used as they notably allow the analysis of molecular structures and in vivo imaging. ${ }^{1-4}$ However, the application of these techniques is severely limited by their inherent low sensitivity. The disadvantages associated with NMR sensitivity can be overcome by making use of hyperpolarization of nuclei in the substance of interest by way of dynamic nuclear polarization (DNP). This technique involves the microwave-promoted transfer of the spin polarization of a polarizing agent (PA, e.g., a radical) to non-zero spin nuclei (e.g. $,{ }^{1} \mathrm{H},{ }^{13} \mathrm{C},{ }^{15} \mathrm{~N},{ }^{31} \mathrm{P}$ ) in its vicinity, allowing the enhancement of the NMR signal of a substance of interest or a higher MRI contrast. ${ }^{1-8}$ The polarization transfer between a PA with unpaired electrons and a target nucleus in the liquid state is governed by the Overhauser effect $(\mathrm{OE}) \cdot{ }^{9,10}$ Thus, hyperpolarization

\footnotetext{
${ }^{a}$ Institute of Physics, Ecole Polytechnique Fédérale de Lausanne (EPFL), Lausanne CH-1015, Switzerland. E-mail: felipe.saenzpadilla@epfl.ch, mika.tamski@epfl.ch, jonas.milani@epfl.ch

${ }^{b}$ Section of Chemistry and Chemical Engineering, Ecole Polytechnique Fédérale de Lausanne (EPFL), Lausanne CH-1015, Switzerland

'Institute of Materials, Ecole Polytechnique Fédérale de Lausanne (EPFL),

Lausanne CH-1015, Switzerland

$\dagger$ Electronic supplementary information (ESI) available: Experimental procedures, electrochemical characterization, mass spectra, EPR spectra, NMR relaxation and saturation characterization. See DOI: 10.1039/d1cc05350g

\# F. S., M. T. and J. M. contributed equally.
}

in solution at room temperature is commonly achieved by Overhauser DNP (O-DNP), where stable radicals such as $(2,2,6,6-$ tetramethylpiperidin-1-yl)oxyl (TEMPO), $\alpha, \gamma$-bisdiphenylene- $\beta$ phenylallyl (BDPA) or 2,2-diphenyl-1-picrylhydrazyl (DPPH) have been used as PAs. ${ }^{11-15}$ One inherent limitation of using such PAs for sensitivity enhancement is that the presence of unpaired electrons in the hyperpolarized solution accelerates the nuclear spin relaxation back to thermal equilibrium, which is the main source of instability. ${ }^{2,4,16-18}$ This problem has prompted substantial research efforts to preserve the hyperpolarized state in order to enable their use as NMR/MRI probes. It has been shown previously that electrochemistry could be used to generate and remove the PA's unpaired electrons on demand in situ, ${ }^{19}$ potentially improving the hyperpolarization lifetime of the mixture. The proof of principle was achieved by using the dication methyl viologen $\left(\mathrm{MV}^{2+}\right.$, 1,1-dimethyl-4,4bipyridinium) as a redox mediator, known for its capacity to readily undergo a reversible reduction into the radical $\mathrm{MV}^{+\bullet} \cdot{ }^{19-22}$ Despite the excellent enhancement obtained with respect to TEMPO, the radical $\mathrm{MV}^{+} \bullet$ is readily oxidized back to $\mathrm{MV}^{2+}$ in presence of oxygen, thus requiring a controlled atmosphere to enable the DNP process. Nowadays, various types of stable radicals are available and have been explored in different applications due to their resilience, but only a few exhibit practical tunability in terms of intrinsic (electro-) chemical properties. ${ }^{23-28}$ In this regard, Blatter-type (benzo[ $e][1,2,4]$ triazinyl) radicals were shown to be highly stable towards air, water and temperature, as well as to provide a versatile molecular scaffold. Since the initial report by Blatter, these organic radicals have seen an increased interest due to their tunable magnetic and chemical characteristics, provided by the highly delocalized SOMO and its sensitivity to substitution of the benzo $[e][1,2,4]$ triazinyl core. ${ }^{27-32}$ To the best of our knowledge, however, they have not yet been applied for DNP. Hence, and in light of recent developments concerning their synthesis and chemical characteristics, ${ }^{27,28,32}$ we aimed to investigate the polarizing and electrochemical properties of some of these radicals in comparison to (4-hydroxy-2,2,6,6tetramethylpiperidin-1-yl)oxyl (TEMPOL), a well-known polarizing 
a)<smiles>O=CN(C1=NN(c2ccccc2)c2ccccc2N1)c1ccccc1</smiles>

1

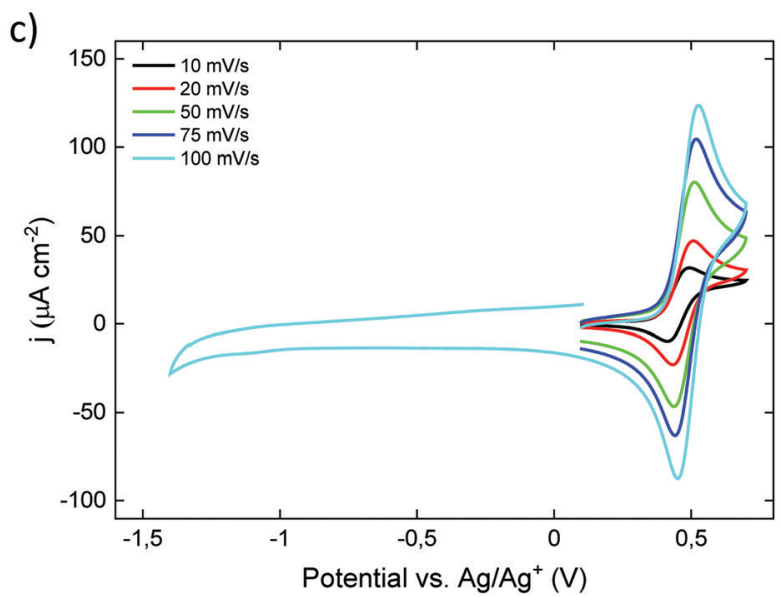

b)<smiles>c1ccc(NC2=NN(c3ccccc3)c3ccccc3N2)cc1</smiles><smiles>CC1(C)CC(O)CC(C)(C)N1[O-]</smiles>

3

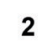

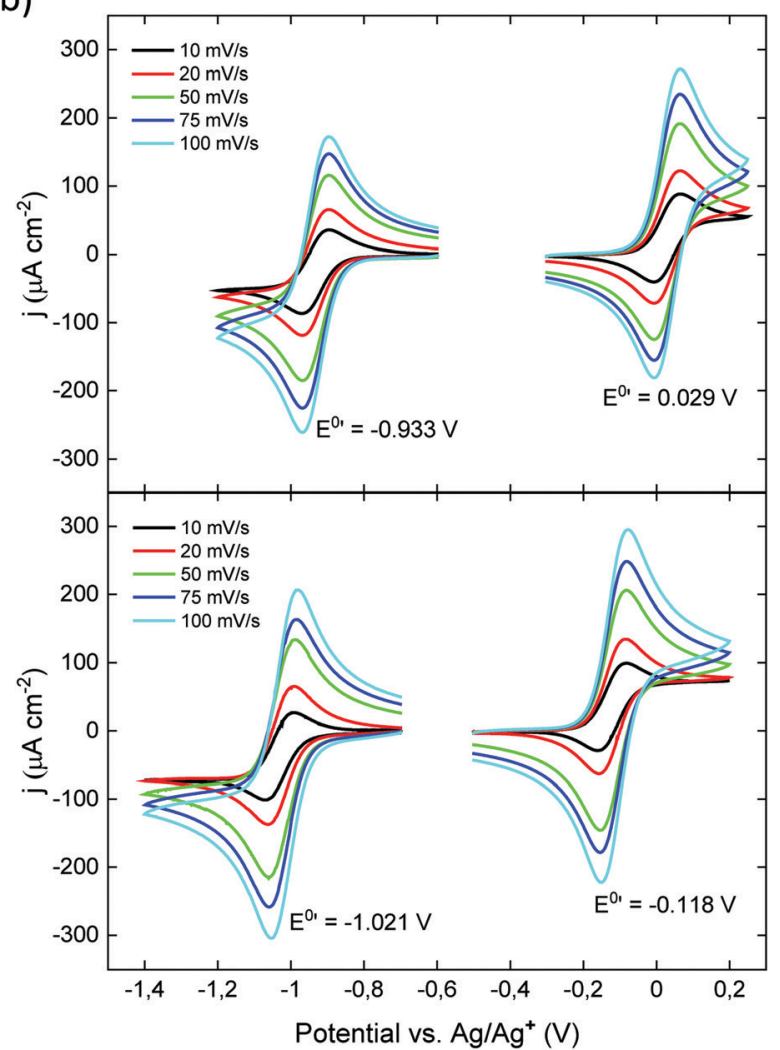

Fig. 1 (a) Molecular structures of the radicals studied. (b) CV behaviour for radicals 1 (top panel) and $\mathbf{2}$ (bottom panel) for the oxidation and reduction of the radical vs scan rate. (c) CV behaviour for TEMPOL (3) for the oxidation of the radical vs. scan rate. The CVs were recorded in a glove box with a glassy carbon working electrode ( $v s . \mathrm{Ag} / \mathrm{Ag}^{+}$) using solutions of the radicals $(1 \mathrm{mM})$ in dry MeCN with $0.1 \mathrm{M}$ TBAP as supporting electrolyte.

agent for DNP. On this account, we report herein the use of Blattertype radicals as polarizing agents for electrochemical DNP. The radicals exhibit superior DNP enhancements with respect to TEMPOL as well as excellent electrochemical stability and reversibility.

The molecular structures of the radicals studied herein are presented in Fig. 1a. The electrochemical behavior of the radicals 1 and 2 in comparison to TEMPOL (3) was assessed by cyclic voltammetry (CV, Fig. 1). The cyclic voltammograms were recorded with $1 \mathrm{mM}$ solutions of the radicals in dry acetonitrile (MeCN) using $0.1 \mathrm{M}$ tetrabutylammonium perchlorate (TBAP) as the supporting electrolyte at different scan rates (see ESI $\dagger$ for details). To accurately determine the peak-to-peak potential separations $\left(\Delta E_{\mathrm{p}}\right)$, the CVs have been corrected for $\mathrm{i} R_{\mathrm{u}}$ drop by compensation of the resistance of each electrochemical cell, typically around $150 \Omega$, determined by measuring the electrochemical impedance spectroscopy (EIS) response of the cell at open-circuit potential for each experiment (see $\mathrm{ESI} \dagger$ ). As shown on Fig. 1b and c, Blatter radicals 1 and 2 exhibit two electrochemical processes whereas TEMPOL displays only one in the same potential range $(-1.4$ to $0.7 \mathrm{~V})$. For each compound, the electrochemical steps proceed by a singleelectron transfer. The voltammograms of the Blatter radicals show a peak to peak potential difference independent of the scan rate and close to the theoretical value of $59 \mathrm{mV}$ for a single reversible electron transfer at $25{ }^{\circ} \mathrm{C}$ (Table 1$) .{ }^{33}$ In addition, the peak current ratios are also close to unity (Table 1 and ESI $\dagger$ Fig. S1) which also confirms the reversibility of the electrochemical processes, in agreement with the literature for these experimental conditions. ${ }^{28}$ The electrochemical reversibility of the different radicals under study is crucial to allow several consecutive DNP processes without significant polarization loss

Table 1 The $E^{0 \prime}, \mathrm{i} P_{\mathrm{A}} / \mathrm{i} P_{\mathrm{C}}$ ratios and $\Delta E_{\mathrm{P}}$ values for the three species studied. All the values are averaged over the 5 scan rates employed, and the respective standard deviations are in brackets (See ESI, Table S1)

\begin{tabular}{llllll}
\hline & Radical 1 & & Radical 2 & \multicolumn{2}{c}{ TEMPOL } \\
\cline { 2 - 3 } & Ox & Red & & Ox & Red \\
\hline$E^{0 \prime}(\mathrm{V})$ & $0.0290\left( \pm 5 \times 10^{-4}\right)$ & $-0.9330\left( \pm 8 \times 10^{-4}\right)$ & & $-0.118( \pm 0.003)$ & $-1.025( \pm 0.005)$ \\
$\mathrm{i} P_{\mathrm{A}} / \mathrm{i} P_{\mathrm{C}}$ & $1.08( \pm 0.03)$ & $0.95( \pm 0.03)$ & & $1.02( \pm 0.03)$ & $0.99( \pm 0.04)$ \\
$\Delta E_{\mathrm{P}}(\mathrm{mV})$ & $69.4( \pm 2.3)$ & $70.0( \pm 1.9)$ & $72.0( \pm 3.4)$ & $74.2( \pm 3.3)$
\end{tabular}


(see below), which is why the reduction of TEMPOL was not considered (see ESI, $\dagger$ Fig. S3). ${ }^{34,35}$ Moreover, the linear dependence of the peak currents $\left(I_{\mathrm{p}}\right)$ as a function of the square root of the scan rate (see ESI, $\dagger$ Fig. S1) revealed the diffusioncontrolled character of the electrochemical processes, ${ }^{36}$ which are therefore compatible with a diffusion-mediated O-DNP. ${ }^{19}$ The same findings were made for TEMPOL (see ESI, $\dagger$ Fig. S1). On account of the electrochemical reversibility of the Blatter radicals, the formal redox potentials $\left(E^{0 \prime}\right)$ of each electrochemical step were assessed by the half sum of the peak potentials. As shown in Table 1, radical 1 presents greater formal oxidation potentials than radical 2 , in accordance to the inductive effect of their respective substituents. Regarding the formal reduction potentials, the difference is less pronounced, probably due to a decreased substituent influence on the anionic species. Through these electrochemical analyses, one can notice the strong influence of the electronic nature of the Blatter substituents on the measured formal potentials as it should be for $\pi$ electron-rich structures. For instance, a correlation between the Hammett constants of several substituents of Blatter radicals and their respective redox potentials was previously reported, which confirms this tendency. ${ }^{28}$ The comparison of steady-state currents between radical 1 and TEMPOL at a $10 \mu \mathrm{m}$ diameter Pt ultramicroelectrode (see ESI, $\dagger$ Fig. S2) yielded diffusion coefficients of $1.7 \times 10^{-5}$ and $7.3 \times 10^{-6} \mathrm{~cm}^{2} \mathrm{~s}^{-1}$, respectively. Thus, we can expect the diffusion rate of the Blatter radicals to be at least two times higher than for a typical nitroxide radical in a polar solvent such as MeCN. Concerning the potential difference between the formal oxidation and reduction potentials for radicals 1 and 2 , values of $0.96 \mathrm{~V}$ and $0.83 \mathrm{~V}$ were obtained, respectively. These values are in agreement with the previous report by Grant et al. ${ }^{32}$ within 10 and $20 \mathrm{mV}$, which we can consider to be satisfactory when accounting for different working electrode materials (Pt instead of glassy carbon) and the inherent precision of cyclic voltammetry (on the order of a few $\mathrm{mV}$ on the peak potential determination). We proceeded with the characterization of the polarizing capabilities of the Blatter radicals. The experiments were performed with $50 \mathrm{mM}$ solutions of TEMPOL and radical 1, and with a saturated (13 $\mathrm{mM}$ ) solution of radical 2 . It should be noted that at these concentrations, the hyperfine structures are no longer visible on the respective EPR spectra (see ESI, $\dagger$ Fig. S6). The ${ }^{1} \mathrm{H}-\mathrm{NMR}$ signal integral was optimized by scanning the frequency of the microwave irradiation, as shown in Fig. 2a, where the respective profiles obtained for radicals $\mathbf{1}$ and $\mathbf{2}$ revealed a similar behaviour. To prevent instability associated with heating, the measurements were performed at low power $(47 \mathrm{~mW})$. As expected for systems where dipolar interactions dominate the polarization transfer between the unpaired electrons and ${ }^{1} \mathrm{H}$ nuclei, the enhancements are negative. ${ }^{10}$ Fig. $2 \mathrm{~b}$ displays the absolute enhancement build-up for radicals $\mathbf{1}$ and 2 as well as for TEMPOL, with maximum absolute enhancements of 53, 64 and 42 respectively. The difference between radical 1 and TEMPOL could be due to their saturation factors, although the peculiar behaviour of radical 2 suggests a different coupling factor and requires further investigation (see ESI, $\dagger$ Fig. S7, S8 and Table S2). ${ }^{37}$ In order to determine if the DNP enhancement could be switched electrochemically and to probe the stability the redox processes under ambient conditions, we submitted radical 1 (50 $\mathrm{mM}$ in $\mathrm{MeCN}$ ) to a series of oxidation and reduction cycles in ambient conditions and measured the enhancement at each redox step (Fig. 3). We started the experiment by oxidizing radical 1 into its diamagnetic, cationic form, which effectively suppressed the DNP enhancement. After each reduction step, which restored the radicals, absolute enhancements in the range 24-30 were obtained. This is consistent with a decreased polarization efficiency in aerated conditions. Nonetheless, we observed that the oxidation was increasingly difficult to accomplish with every iteration, probably due to slow fouling of the working electrode which required increasing the oxidation potential applied to the electrode for a longer period of time for each electrochemical
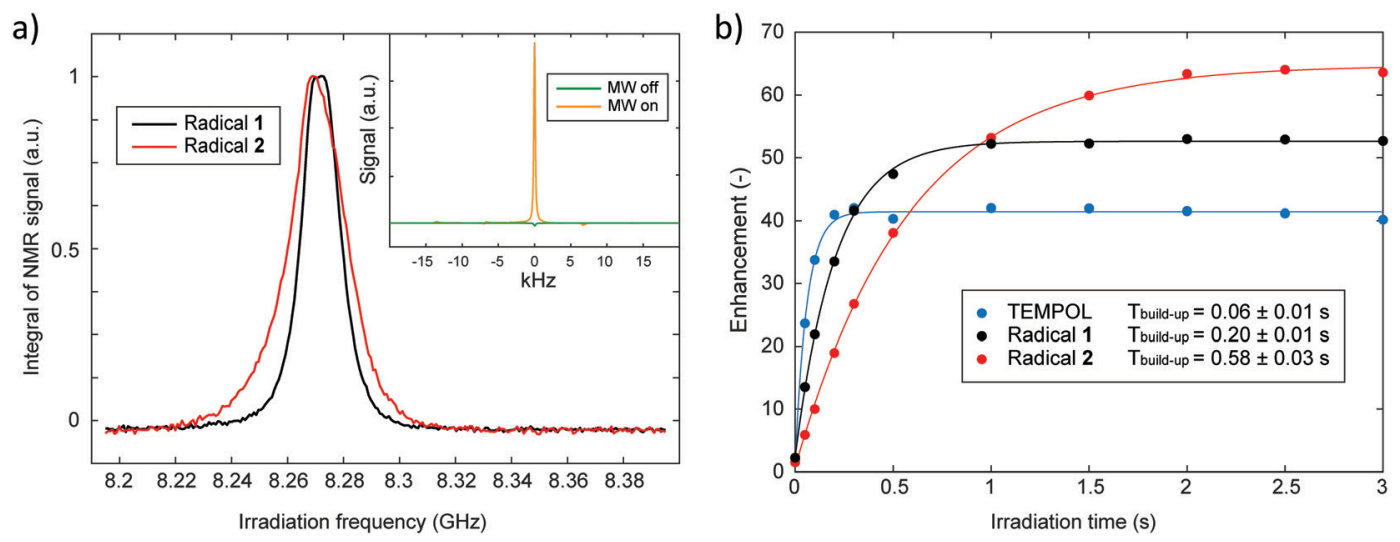

Fig. 2 (a) DNP microwave frequency scans of the solvent ${ }^{1} \mathrm{H}$ signal integral. The spectra were recovered with $30 \mathrm{NMR}$ scans and 250 points with $47 \mathrm{~mW}$ of irradiation. Inset: Typical ${ }^{1} \mathrm{H}-\mathrm{NMR}$ spectrum at $12.5 \mathrm{MHz}$ with (orange line) and without (green line) microwave irradiation at $8.269 \mathrm{GHz}$ and $8.270 \mathrm{GHz}$ for the Blatter radical solutions. The thermal equilibrium (microwave off) is an average of 1000 scans and the DNP signal (microwave on) is an average of 30 scans. (b) Build-up magnetization for radicals 1 (black circles), 2 (red circles) and TEMPOL (blue circles), with maximum absolute enhancements of 53, 64 and 42 respectively. Build-up times were extracted from fitting. The pulse sequence contains a saturation recovery to start the measurement. 


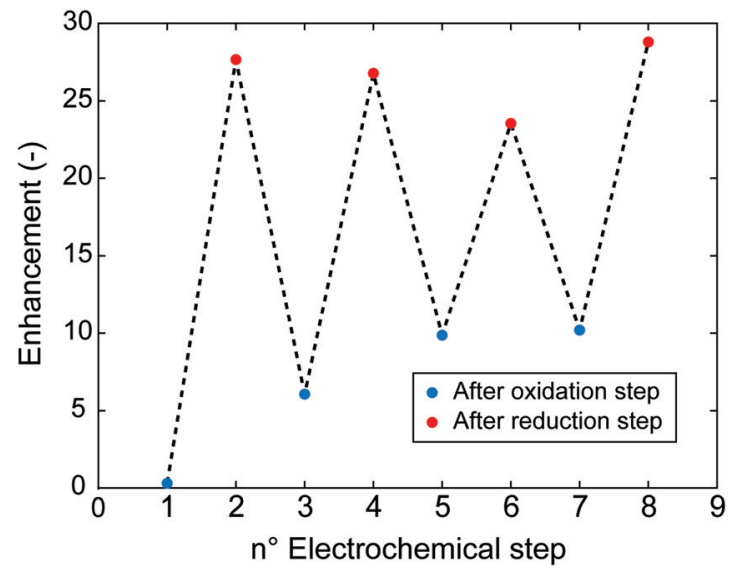

Fig. 3 Electrochemical redox switching of the O-DNP absolute enhancement. The enhanced ${ }^{1} \mathrm{H}-\mathrm{NMR}$ solvent signals are measured using a solution of radical $1(50 \mathrm{mM})$ in $\mathrm{MeCN}$ with TBAHFP as supporting electrolyte (see ESI $\dagger$ for details).

step, suggesting that an optimization of the electrochemical setup is necessary to improve the reversibility of the process. Despite this technical difficulty associated with the electrolytic cell as a whole, radical 1 exhibited a good stability in the experiment timeframe under ambient conditions.

In conclusion, we demonstrate herein that Blatter-type radicals such as 1 and 2 can be used as a switchable polarizing agent for Overhauser DNP, with enhancements exceeding the values typically obtained with the common polarizing agent TEMPOL. In addition to an excellent stability of the employed Blatter-type radicals, they are even more promising polarizing agents when one considers their straightforward (electro-) chemical tunability, as the versatile benzo[e][1,2,4]triazinyl scaffold tolerates a wide range of chemical transformations such as the addition of extended $\pi$-systems, strongly inductive chemical groups or the synthesis of diradicaloids. ${ }^{28}$ Furthermore, we show that radical 1 can be electrochemically generated and removed in situ, potentially paving the way towards applications different from NMR/MRI, such as antioxidant probes or batteries and super capacitors.

The manuscript was written through contributions of all authors. All authors have given approval to the final version of the manuscript.

Funding This project was funded by Innosuisse, Innovation project no. 40981.1 IP-ENG.

\section{Conflicts of interest}

There are no conflicts to declare.

\section{Notes and references}

1 A. Naito, Experimental Approaches of NMR Spectroscopy: Methodology and Application to Life Science and Materials Science, Springer, Singapore, 2018.

2 J. H. Ardenkjaer-Larsen, J. Magn. Reson., 2016, 264, 3-12.
3 R. W. Hooper, B. A. Klein and V. K. Michaelis, Chem. Mater., 2020, 32, 4425-4430.

4 D. Gajan, A. Bornet, B. Vuichoud, J. Milani, R. Melzi, H. A. van Kalkeren, L. Veyre, C. Thieuleux, M. P. Conley, W. R. Gruning, M. Schwarzwalder, A. Lesage, C. Coperet, G. Bodenhausen, L. Emsley and S. Jannin, Proc. Natl. Acad. Sci. U. S. A., 2014, 111, 14693-14697.

5 J. H. Ardenkjaer-Larsen, B. Fridlund, A. Gram, G. Hansson, L. Hansson, M. H. Lerche, R. Servin, M. Thaning and K. Golman, Proc. Natl. Acad. Sci. U. S. A., 2003, 100, 10158-10163.

6 G. Casano, H. Karoui and O. Ouari, eMagRes, 2018, 7, 195-207.

7 A. W. Overhauser, Phys. Rev., 1953, 92, 411-415.

8 T. R. Carver and C. P. Slichter, Phys. Rev., 1953, 92, 212-213.

9 N. Abhyankar and V. Szalai, J. Phys. Chem. B, 2021, 125, 5171-5190.

10 B. Corzilius, Annu. Rev. Phys. Chem., 2020, 71, 143-170.

11 P. Höfer, G. Parigi, C. Luchinat, P. Carl, G. Guthausen, M. Reese, T. Carlomagno, C. Griesinger and M. Bennati, J. Am. Chem. Soc., 2008, 130, 3254-3255.

12 N. M. Loening, M. Rosay, V. Weis and R. G. Griffin, J. Am. Chem. Soc., 2002, 124, 8808-8809.

13 H. G. Beljers, L. van der Kint and J. S. van Wieringen, Phys. Rev., 1954, 95, 1683.

14 M. D. Lingwood and S. Han, Annual Reports on NMR Spectroscopy, Elsevier, 2011, vol. 73, pp. 83-126.

15 W. Müller-Warmuth and K. Meise-Gresch, Advances in Magnetic and Optical Resonance, Elsevier, 1983, vol. 11, pp. 1-45.

16 D. Gajan, M. Schwarzwälder, M. P. Conley, W. R. Grüning, A. J. Rossini, A. Zagdoun, M. Lelli, M. Yulikov, G. Jeschke, C. Sauvée, O. Ouari, P. Tordo, L. Veyre, A. Lesage, C. Thieuleux, L. Emsley and C. Copéret, J. Am. Chem. Soc., 2013, 135, 15459-15466.

17 X. Ji, A. Bornet, B. Vuichoud, J. Milani, D. Gajan, A. J. Rossini, L. Emsley, G. Bodenhausen and S. Jannin, Nat. Commun., 2017, 8, 13975.

18 A. Capozzi, T. Cheng, G. Boero, C. Roussel and A. Comment, Nat. Commun., 2017, 8, 15757.

19 M. Tamski, J. Milani, C. Roussel and J.-P. Ansermet, Phys. Chem. Chem. Phys., 2020, 22, 17769-17776.

20 P. K. Bhowmik, H. Han, J. J. Cebe, R. A. Burchett and A. M. Sarker, J. Polym. Sci., Part A: Polym. Chem., 2002, 40, 659-674.

21 J. Ding, C. Zheng, L. Wang, C. Lu, B. Zhang, Y. Chen, M. Li, G. Zhai and X. Zhuang, J. Mater. Chem. A, 2019, 7, 23337-23360.

22 K. Madasamy, D. Velayutham, V. Suryanarayanan, M. Kathiresan and K.-C. Ho, J. Mater. Chem. C, 2019, 7, 4622-4637.

23 Q. Z. Ni, F. Yang, T. V. Can, I. V. Sergeyev, S. M. D'Addio, S. K. Jawla, Y. Li, M. P. Lipert, W. Xu, R. T. Williamson, A. Leone, R. G. Griffin and Y. Su, J. Phys. Chem. B, 2017, 121, 8132-8141.

24 Y. Su, L. Andreas and R. G. Griffin, Annu. Rev. Biochem., 2015, 84, 465-497.

25 P. M. Lahti, Advances in Physical Organic Chemistry, Elsevier, 2011, vol. 45, pp. 93-169.

26 K. Zhang, M. J. Monteiro and Z. Jia, Polym. Chem., 2016, 7, 5589-5614.

27 Y. Ji, L. Long and Y. Zheng, Mater. Chem. Front., 2020, 4, 3433-3443.

28 F. J. M. Rogers, P. L. Norcott and M. L. Coote, Org. Biomol. Chem., $2020,18,8255-8277$.

29 H. M. Blatter and H. Lukaszewski, Tetrahedron Lett., 1968, 22, 2701-2705.

30 P. Bartos, B. Anand, A. Pietrzak and P. Kaszyński, Org. Lett., 2020, 22, 180-184.

31 A. Bodzioch, M. Zheng and P. Kaszyns, J. Org. Chem., 2014, 17.

32 J. A. Grant, Z. Lu, D. E. Tucker, B. M. Hockin, D. S. Yufit, M. A. Fox, R. Kataky, V. Chechik and A. C. O'Donoghue, Nat. Commun., 2017, 8, 15088.

33 J. Daschbach, D. Blackwood, J. W. Pons and S. Pons, J. Electroanal. Chem. Interfacial Electrochem., 1987, 237, 269-273.

34 T. Suga, Y.-J. Pu, S. Kasatori and H. Nishide, Macromolecules, 2007, 40, 3167-3173.

35 X. Wei, W. Pan, W. Duan, A. Hollas, Z. Yang, B. Li, Z. Nie, J. Liu, D. Reed, W. Wang and V. Sprenkle, ACS Energy Lett., 2017, 2, 2187-2204.

36 N. Elgrishi, K. J. Rountree, B. D. McCarthy, E. S. Rountree, T. T. Eisenhart and J. L. Dempsey, J. Chem. Educ., 2018, 95, 197-206.

37 M. Levien, M. Reinhard, M. Hiller, I. Tkach, M. Bennati and T. Orlando, Phys. Chem. Chem. Phys., 2021, 23, 4480-4485. 